\title{
THE MOORE-PENROSE INVERSE OF A LINEAR COMBINATION OF COMMUTING GENERALIZED AND HYPERGENERALIZED PROJECTORS*
}

\author{
MARINA TOŠIĆ ${ }^{\dagger}$, DRAGANA S. CVETKOVIĆ-ILIĆ ${ }^{\dagger}$, AND CHUNYUAN DENG
}

\begin{abstract}
In this paper, some representations for the Moore-Penrose inverse of a linear combination of generalized and hypergeneralized projectors are found. Also, the invertibility for some linear combinations of commuting generalized and hypergeneralized projectors is considered.
\end{abstract}

Key words. Idempotent, Projector, Generalized projector, Hypergeneralized projector, MoorePenrose inverse.

AMS subject classifications. 15A09.

1. Introduction. Let $\mathbb{C}^{n \times m}$ denote the set of all $n \times m$ complex matrices. The symbols $A^{*}, \mathcal{R}(A), \mathcal{N}(A)$, and $\operatorname{rank}(A)$ will denote the conjugate transpose, the range (column space), the null space, and the rank of a matrix $A$, respectively. By $\mathbb{C}_{r}^{n \times n}$ we will denote the set of all matrices from $\mathbb{C}^{n \times n}$ with a rank $r$. The Moore-Penrose inverse of $A$, is the unique matrix $A^{\dagger}$ satisfying the equations
(1) $A A^{\dagger} A=A$,
(2) $A^{\dagger} A A^{\dagger}=A^{\dagger}$,
(3) $A A^{\dagger}=\left(A A^{\dagger}\right)^{*}$,
(4) $A^{\dagger} A=\left(A^{\dagger} A\right)^{*}$.

For a square matrix $A$ there exists a unique reflexive generalized inverse of $A$ which commutes with $A$ if and only if $A$ is of the index 1, that is, $\operatorname{rank}(A)=\operatorname{rank}\left(A^{2}\right)$ ([4], Theorem 1). This generalized inverse is called the group inverse of $A$ and is denoted by $A^{\sharp}$.

$I_{n}$ will denote the identity matrix of order $n$ while $0_{s, s}$ will denote the nullmatrix of order $s$. We use the notations $C_{n}^{P}, C_{n}^{O P}, C_{n}^{E P}, C_{n}^{G P}$, and $C_{n}^{H G P}$ for the subsets of $\mathbb{C}^{n \times n}$ consisting of projectors (idempotent matrices), orthogonal projectors (Hermitian idempotent matrices ), EP (range-Hermitian) matrices, generalized, and

${ }^{*}$ Received by the editors on August 14, 2011. Accepted for publication on October 24, 2011. Handling Editor: Oskar Maria Baksalary.

${ }^{\dagger}$ University of Niš, Faculty of Sciences and Mathematics, Serbia (marinatosic@ymail.com, dragana@pmf.ni.ac.rs). Supported by grant no. 174007 of the Ministry of Science and Technological Development, Republic of Serbia.

${ }^{\ddagger}$ School of Mathematical Sciences, South China Normal University, Guangzhou 510631, P.R. China (cydeng@scnu.edu.cn). Supported by the National Natural Science Foundation of China under grant no. 11171222 and the Doctoral Program of the Ministry of Education under grant no. 20094407120001. 
hypergeneralized projectors, respectively, i.e.,

$$
\begin{aligned}
& C_{n}^{P}=\left\{A \in \mathbb{C}^{n \times n}: A^{2}=A\right\}, \\
& C_{n}^{O P}=\left\{A \in \mathbb{C}^{n \times n}: A^{2}=A=A^{*}\right\}, \\
& C_{n}^{E P}=\left\{A \in \mathbb{C}^{n \times n}: \mathcal{R}(A)=\mathcal{R}\left(A^{*}\right)\right\}=\left\{A \in \mathbb{C}^{n \times n}: A A^{\dagger}=A^{\dagger} A\right\}, \\
& C_{n}^{G P}=\left\{A \in \mathbb{C}^{n \times n}: A^{2}=A^{*}\right\}, \\
& C_{n}^{H G P}=\left\{A \in \mathbb{C}^{n \times n}: A^{2}=A^{\dagger}\right\} .
\end{aligned}
$$

The concepts of generalized and hypergeneralized projectors were introduced by Groß and Trenkler [9] who presented interesting properties of the classes of generalized and hypergeneralized projectors. Very interesting results concerning generalized and hypergeneralized projectors can be found in the papers of J.K. Baksalary, O.M. Baksalary, X. Liu, and G. Trenkler [2], O.M. Baksalary [1], J.K. Baksalary, O.M. Baksalary, and J. Groß [3], J. Benítez and N. Thome [6], and G.W. Stewart [11].

In this paper, we give the form for the Moore-Penrose inverse, i.e., the group inverse of a linear combination $c_{1} A+c_{2} B$ of two commuting generalized or hypergeneralized projectors. Also, we studied the nonsingularity of $c_{1} A+c_{2} B$ and $c_{1} A+c_{2} B+c_{3} C$, where $A, B$ and $C$ are commuting generalized or hypergeneralized projectors under various conditions.

2. The Moore-Penrose inverse and the invertibility of a linear combination of commuting generalized or hypergeneralized projections. J.K. Baksalary, O.M. Baksalary, X. Liu, and G. Trenkler [2], proved that any generalized projector $A \in \mathbb{C}_{r}^{n \times n}$ can be represented by

$$
A=U\left[\begin{array}{cc}
K & 0 \\
0 & 0
\end{array}\right] U^{*}
$$

where $U \in C^{n \times n}$ is unitary and $K \in \mathbb{C}^{r \times r}$ is such that $K^{3}=I_{r}$ and $K^{*}=K^{-1}$. Any hypergeneralized projector $A \in \mathbb{C}_{r}^{n \times n}$ has a form

$$
A=U\left[\begin{array}{cc}
\Sigma K & 0 \\
0 & 0
\end{array}\right] U^{*}
$$

where $U \in C^{n \times n}$ is unitary, $\Sigma=\operatorname{diag}\left(\sigma_{1} I_{r_{1}}, \ldots, \sigma_{t} I_{r_{t}}\right)$ is a diagonal matrix of singular values of $\mathrm{A}, \sigma_{1}>\sigma_{2}>\cdots>\sigma_{t}>0, r_{1}+r_{2}+\cdots+r_{t}=r$ and $K \in \mathbb{C}^{r \times r}$ satisfies $(\Sigma K)^{3}=I_{r}$ and $K K^{*}=I_{r}$.

There are also some other very useful representations for generalized and hypergeneralized projectors. By using the fact that any generalized projector $A \in \mathbb{C}_{r}^{n \times n}$ is a normal matrix, by the spectral theorem we have that $A=U \operatorname{diag}\left(\lambda_{1}, \lambda_{2}, \ldots, \lambda_{n}\right) U^{*}$, 
where $U$ is a unitary matrix and $\lambda_{j}, j=\{1, \ldots, n\}$ are the eigenvalues of $A$. By $[6$, Theorem 2.1], we have that $\lambda_{j} \in\{0,1, \omega, \bar{\omega}\}, j=\{1, \ldots, n\}$, where $\omega=\exp (2 \pi i / 3)$. Hence,

$$
A \in C_{n}^{G P} \Leftrightarrow A=U \operatorname{diag}\left(\lambda_{1}, \lambda_{2}, \ldots, \lambda_{n}\right) U^{*}
$$

where $U^{*}=U^{-1}$ and $\lambda_{j} \in\{0,1, \omega, \bar{\omega}\}, j=\{1, \ldots, n\}, \omega=\exp (2 \pi i / 3)$.

Similarly, for $A \in C_{n}^{H G P}$ using the fact that $A$ is EP-matrix, by [7, Theorem 4.3.1] we can conclude that

$$
A \in C_{n}^{H G P} \Leftrightarrow A=U(K \oplus 0) U^{*}
$$

where $U \in \mathbb{C}^{n \times n}$ is a unitary matrix and $K \in \mathbb{C}^{r \times r}$ is nonsingular such that $K^{3}=I_{r}$, where $r=\operatorname{rank}(A)$.

From the above representations it is obvious that any generalized projector is a hypergeneralized projector.

The following fact will be used very often:

If $X, Y \in \mathbb{C}^{n \times n}$ and $c_{1}, c_{2} \in \mathbb{C}$, then

$$
\begin{aligned}
X^{3}=Y^{3}= & I_{n}, X Y=Y X \Rightarrow \\
& \left(c_{1} X+c_{2} Y\right)\left(c_{1}^{2} X^{2}-c_{1} c_{2} X Y+c_{2}^{2} Y^{2}\right)=\left(c_{1}^{3}+c_{2}^{3}\right) I_{n} .
\end{aligned}
$$

In this section, we first present the form for the Moore-Penrose inverse, i.e., the group inverse of $c_{1} A+c_{2} B$, where $A, B$ are two commuting generalized or hypergeneralized projectors and $c_{1}, c_{2} \in \mathbb{C} \backslash\{0\}$ and $c_{1}^{3}+c_{2}^{3} \neq 0$.

Theorem 2.1. Let $A \in \mathbb{C}^{n \times n}$ and $B \in \mathbb{C}^{n \times n}$ be commuting generalized or hypergeneralized projectors, and let $c_{1}, c_{2} \in \mathbb{C} \backslash\{0\}$ and $c_{1}^{3}+c_{2}^{3} \neq 0$. Then

$$
\begin{aligned}
\left(c_{1} A+c_{2} B\right)^{\dagger}= & \frac{1}{c_{1}^{3}+c_{2}^{3}}\left(c_{1}^{2} A^{2} B^{3}-c_{1} c_{2} A B+c_{2}^{2} A^{3} B^{2}\right)+\frac{1}{c_{1}} A^{2}\left(I_{n}-B^{3}\right) \\
& +\frac{1}{c_{2}} B^{2}\left(I_{n}-A^{3}\right) .
\end{aligned}
$$

Furthermore, $c_{1} A+c_{2} B$ is nonsingular if and only if $n=\operatorname{rank}(A)+\operatorname{rank}(B)-$ $\operatorname{rank}(A B)$ and in this case $\left(c_{1} A+c_{2} B\right)^{-1}$ is given by $(2.2)$.

Proof. Since $A$ and $B$ are two commuting EP-matrices, by [5, Corollary 3.9], we have that

$$
A=U\left(A_{1} \oplus A_{2} \oplus 0_{t, t} \oplus 0\right) U^{*}, \quad B=U\left(B_{1} \oplus 0_{s, s} \oplus B_{2} \oplus 0\right) U^{*}
$$


where $A_{1}, B_{1} \in \mathbb{C}^{r \times r}, A_{2} \in \mathbb{C}^{s \times s}, B_{2} \in \mathbb{C}^{t \times t}$ are nonsingular and $A_{1} B_{1}=B_{1} A_{1}$. If in addition $A$ and $B$ are hypergeneralized projectors (the following reasoning works if $A, B$ are generalized projectors), then $A_{1}^{3}=B_{1}^{3}=I_{r}, A_{2}^{3}=I_{s}$, and $B_{2}^{3}=I_{t}$. Since

$$
c_{1} A+c_{2} B=U\left(\left(c_{1} A_{1}+c_{2} B_{1}\right) \oplus c_{1} A_{2} \oplus c_{2} B_{2} \oplus 0\right) U^{*},
$$

we can use (2.1) to get the expression for $\left(c_{1} A+c_{2} B\right)^{\dagger}$. Thus, by (2.1) we get that $c_{1} A_{1}+c_{2} B_{1}$ is nonsingular and that

$$
\left(c_{1} A_{1}+c_{2} B_{1}\right)^{-1}=\frac{1}{c_{1}^{3}+c_{2}^{3}}\left(c_{1}^{2} A_{1}^{2}-c_{1} c_{2} A_{1} B_{1}+c_{2}^{2} B_{1}^{2}\right) .
$$

Now, using that

$$
I_{n}-A^{3}=U\left(0 \oplus 0 \oplus I_{t} \oplus I_{n-(r+t+s)}\right) U^{*}, \quad A^{3} B^{3}=U\left(I_{r} \oplus 0 \oplus 0 \oplus 0\right) U^{*}
$$

and

$$
I_{n}-B^{3}=U\left(0 \oplus I_{s} \oplus 0 \oplus I_{n-(r+t+s)}\right) U^{*}
$$

we have

$$
\begin{aligned}
\left(c_{1} A+c_{2} B\right)^{\dagger}= & U\left(\left(c_{1} A_{1}+c_{2} B_{1}\right)^{-1} \oplus \frac{1}{c_{1}} A_{2}^{2} \oplus \frac{1}{c_{2}} B_{2}^{2} \oplus 0\right) U^{*} \\
= & \frac{1}{c_{1}^{3}+c_{2}^{3}}\left(c_{1}^{2} A^{2}-c_{1} c_{2} A B+c_{2}^{2} B^{2}\right) A^{3} B^{3} \\
& +\frac{1}{c_{1}} A^{2}\left(I_{n}-B^{3}\right)+\frac{1}{c_{2}} B^{2}\left(I_{n}-A^{3}\right) .
\end{aligned}
$$

Since $A^{4}=A$ and $B^{4}=B$, we get that (2.2) holds. Also, it is evident that $\operatorname{rank}(A)=$ $r+s, \operatorname{rank}(B)=r+t$ and $\operatorname{rank}(A B)=r$. So, the last summand in the direct sum of (2.3) does not appear if and only if $n=\operatorname{rank}(A)+\operatorname{rank}(B)-\operatorname{rank}(A B)$, which is a necessary and sufficient condition for the invertibility of $c_{1} A+c_{2} B$.

As a corollary, we get that in the case when $A$ is generalized or hypergeneralized projector and $c_{1}, c_{2} \in \mathbb{C}, c_{1} \neq 0, c_{1}^{3}+c_{2}^{3} \neq 0$, a linear combination $c_{1} I_{n}+c_{2} A$ is always nonsingular.

ThEOREM 2.2. Let $A \in \mathbb{C}^{n \times n}$ be a generalized or hypergeneralized projector, $c_{1}, c_{2} \in \mathbb{C}, c_{1} \neq 0, c_{1}^{3}+c_{2}^{3} \neq 0$. Then $c_{1} I_{n}+c_{2} A$ is nonsingular and

$$
\left(c_{1} I_{n}+c_{2} A\right)^{-1}=\frac{1}{c_{1}^{3}+c_{2}^{3}}\left(c_{1}^{2} A^{3}-c_{1} c_{2} A+c_{2}^{2} A^{2}\right)+\frac{1}{c_{1}}\left(I_{n}-A^{3}\right) .
$$

Let $\mathcal{G} \subset C_{n}^{G P}$ denote a commuting family of generalized projectors and $\mathcal{H} \subset$ $C_{n}^{H G P}$ denote a commuting family of hypergeneralized projectors, i.e., an infinite 
set of generalized projectors or hypergeneralized projectors in which each pair in the set commutes under multiplication. If we consider a finite commuting family $\left\{A_{i}\right\}_{i=1}^{m}$ where all of the members are generalized or hypergeneralized projectors, then $\prod_{i=1}^{m} A_{i}^{k_{i}}$, where $k_{1}, \ldots, k_{m} \in \mathbb{N}$, is also a generalized or hypergeneralized projector. Hence, we have the following result:

Proposition 2.3. Let all of $A_{i} \in \mathbb{C}^{n \times n}, i=\{1, \ldots, m\}$ be commuting generalized or hypergeneralized projectors, $c_{1}, c_{2} \in \mathbb{C}, c_{1} \neq 0, c_{1}^{3}+c_{2}^{3} \neq 0$ and $k_{1}, \ldots, k_{m} \in \mathbb{N}$ . Then $c_{1} I_{n}+c_{2} \prod_{i=1}^{m} A_{i}^{k_{i}}$ is nonsingular.

With the additional requirements of Theorem 2.1 it is possible to give a more precise form of Moore-Penrose inverse, i.e., the group inverse.

Corollary 2.4. Let $c_{1}, c_{2} \in \mathbb{C} \backslash\{0\}$. If $A, B$ are commuting generalized or hypergeneralized projectors such that $A B=0$, then

$$
\left(c_{1} A+c_{2} B\right)^{\dagger}=\frac{1}{c_{1}} A^{2}+\frac{1}{c_{2}} B^{2} .
$$

In the next result, we present the form of Moore-Penrose inverse, i.e., the group inverse of $c_{1} A^{m}+c_{2} A^{k}$, where $m, k \in \mathbb{N}$ and $A$ is a generalized or hypergeneralized projector. It is a corollary of Theorem 2.1 .

Corollary 2.5. Let $A \in \mathbb{C}_{r}^{n \times n}$ be a generalized or hypergeneralized projector and let $c_{1}, c_{2} \in \mathbb{C}, c_{1}^{3}+c_{2}^{3} \neq 0$ and $m, k \in \mathbb{N}$. Then

$$
\left(c_{1} A^{m}+c_{2} A^{k}\right)^{\dagger}=\frac{1}{c_{1}^{3}+c_{2}^{3}}\left(c_{1}^{2} A^{2 m}-c_{1} c_{2} A^{m+k}+c_{2} A^{2 k}\right),
$$

where $A^{t}=\left\{\begin{array}{l}A^{3}, \quad t \equiv_{3} 0, \\ A, \quad t \equiv_{3} 1 \\ A^{2}, \quad t \equiv_{3} 2\end{array}\right.$. Furthermore, $c_{1} A^{m}+c_{2} A^{k}$ is nonsingular if and only if $A$ is nonsingular and in this case the inverse of $c_{1} A^{m}+c_{2} A^{k}$ is given by

$$
\left(c_{1} A^{m}+c_{2} A^{k}\right)^{-1}=\frac{1}{c_{1}^{3}+c_{2}^{3}}\left(c_{1}^{2} A^{p}-c_{1} c_{2} A^{q}+c_{2} A^{r}\right),
$$

where $2 m \equiv_{3} p, m+k \equiv_{3} q$ and $2 k \equiv_{3} r$.

Proof. It follows by Theorem 2.1 and the fact that $\operatorname{rank}\left(A^{p}\right)=\operatorname{rank}(A)$, for any $p \in \mathbb{N}$.

As a corollary we get a result from [2].

Corollary 2.6. [2] Let $A \in \mathbb{C}_{r}^{n \times n}$ be a generalized projector and let $c_{1}, c_{2} \in \mathbb{C}$, $c_{1}^{3}+c_{2}^{3} \neq 0$. Then

$$
\left(c_{1} A+c_{2} A^{*}\right)^{\dagger}=\frac{1}{c_{1}^{3}+c_{2}^{3}}\left(c_{1}^{2} A^{2}-c_{1} c_{2} A^{3}+c_{2}^{2} A\right) .
$$


Let us recall that for the matrices $A, B \in \mathbb{C}^{n \times m}$, a matrix $A$ is less than or equal to $B$ with respect to the star partial ordering, denoted by $A \stackrel{*}{\leq} B$ [8], if

$$
A^{*} A=A^{*} B \text { and } A A^{*}=B A^{*} \text {. }
$$

If $A \in C_{n}^{E P}$, then for any $B \in \mathbb{C}^{n \times n}$,

$$
A \stackrel{*}{\leq} B \Leftrightarrow A B=A^{2}=B A
$$

In the next theorem, we present the form of Moore-Penrose inverse, i.e., the group inverse of $c_{1} A^{m}+c_{2} B^{k}$ under the condition that $A, B$ are generalized projectors and $A B=B A=A^{2}$. Remark that the same result holds if we suppose that $A, B$ are generalized projectors such that $B-A \in C_{n}^{G P}$; or $A \in C_{n}^{E P}, B \in C_{n}^{H G P}$ such that $A \stackrel{*}{\leq} B$.

TheOREM 2.7. Let $c_{1}, c_{2} \in \mathbb{C}, c_{2} \neq 0, c_{1}^{3}+c_{2}^{3} \neq 0$ and $m, k \in \mathbb{N}$. If $A \in \mathbb{C}^{n \times n}$ and $B \in \mathbb{C}^{n \times n}$ are hypergeneralized projectors such that $A B=B A=A^{2}$, then

$$
\left(c_{1} A^{m}+c_{2} B^{k}\right)^{\dagger}=\frac{1}{c_{1}^{3}+c_{2}^{3}}\left(c_{1}^{2} A^{2 m}-c_{1} c_{2} A^{m+k}+c_{2}^{2} A^{2 k}\right)+\frac{1}{c_{2}} B^{2 k}\left(I_{n}-A^{3}\right)
$$

where $A^{t}=\left\{\begin{array}{ll}A^{3}, & t \equiv_{3} 0 \\ A, & t \equiv_{3} 1 \\ A^{2}, & t \equiv_{3} 2\end{array}\right.$ and $B^{s}=\left\{\begin{array}{ll}B^{3}, & s \equiv_{3} 0 \\ B, & s \equiv_{3} 1 \\ B^{2}, & s \equiv_{3} 2\end{array}\right.$.

Proof. By [5, Corollary 3.9] and the fact that $A B=B A=A^{2}$, we have that

$$
A=U\left(A_{1} \oplus 0_{t, t} \oplus 0\right) U^{*}, \quad B=U\left(B_{1} \oplus B_{2} \oplus 0\right) U^{*},
$$

where $A_{1}, B_{1} \in \mathbb{C}^{r \times r}, B_{2} \in \mathbb{C}^{t \times t}$ are nonsingular and $A_{1} B_{1}=B_{1} A_{1}=A_{1}^{2}$. Evidently $A_{1}=B_{1}$. If in addition $A$ and $B$ are hypergeneralized projectors, then $A_{1}^{3}=I_{r}$ and $B_{2}^{3}=I_{t}$. Hence,

$$
c_{1} A^{m}+c_{2} B^{k}=U\left(\left(c_{1} A_{1}^{m}+c_{2} A_{1}^{k}\right) \oplus c_{2} B_{2}^{k} \oplus 0\right) U^{*} .
$$

By (2.1) we get that $c_{1} A_{1}^{m}+c_{2} A_{1}^{k}$ is nonsingular and that

$$
\left(c_{1} A_{1}^{m}+c_{2} A_{1}^{k}\right)^{-1}=\frac{1}{c_{1}^{3}+c_{2}^{3}}\left(c_{1}^{2} A_{1}^{2 m}-c_{1} c_{2} A_{1}^{m+k}+c_{2}^{2} A_{1}^{2 k}\right) .
$$

Now, by using that

$$
A^{3}=U\left(I_{r} \oplus 0 \oplus 0\right) U^{*}, \quad B^{3}-A^{3}=U\left(0 \oplus I_{t} \oplus 0\right) U^{*},
$$

we have that (2.4) holds. $\square$ 
REMARK 2.8. If $A \in C_{n}^{E P}, B \in C_{n}^{H G P}$, and $A \stackrel{*}{\leq} B$, we can conclude that $B-A$ is a hypergeneralized projector. If $A$ and $B$ are hypergeneralized projectors, then $A \stackrel{*}{\leq} B$ or $A B=A^{2}=B A$ is sufficient for $B-A$ to be a hypergeneralized projector $[9]$.

TheOREM 2.9. Let $A \in \mathbb{C}_{r}^{n \times n}$ and $B \in \mathbb{C}^{n \times n}$ be commuting hypergeneralized projectors. Let $c_{1}, c_{2} \in \mathbb{C} \backslash\{0\}, c_{1}^{3}+c_{2}^{3} \neq 0$ and $m, k, l \in \mathbb{N}$. Then

$$
\begin{aligned}
{\left[A^{m}\left(c_{1} A^{k}+c_{2} B^{l}\right)\right]^{\dagger}=} & \frac{1}{c_{1}^{3}+c_{2}^{3}}\left(c_{1}^{2} A^{2(m+k)} B^{3}-c_{1} c_{2} A^{(2 m+k)} B^{l}+c_{2}^{2} A^{2 m} B^{2 l}\right) \\
& +\frac{1}{c_{1}} A^{2(m+k)}\left(I_{n}-B^{3}\right),
\end{aligned}
$$

where $A^{t}=\left\{\begin{array}{ll}A^{3}, & t \equiv_{3} 0 \\ A, & t \equiv_{3} 1 \\ A^{2}, & t \equiv_{3} 2\end{array}\right.$ and $B^{s}=\left\{\begin{array}{ll}B^{3}, & s \equiv_{3} 0 \\ B, & s \equiv_{3} 1 \\ B^{2}, & s \equiv_{3} 2\end{array}\right.$.

Proof. The proof is similar to the proof of the Theorem 2.1. $\square$

The following theorem presents a necessary and sufficient condition for the invertibility of $c_{1} A+c_{2} B+c_{3} C$ in the case when $A, B, C$ are commuting hypergeneralized projectors such that $B C=0$ and $c_{1}, c_{2}, c_{3} \in \mathbb{C} \backslash\{0\}, c_{1}^{3}+c_{2}^{3} \neq 0, c_{1}^{3}+c_{3}^{3} \neq 0$. Remark that the same result holds if we suppose that $A, B, C \in \mathcal{G}$ such that $B+C \in C_{n}^{G P}$, or when $A, B, C \in \mathcal{H}$ such that $B \stackrel{*}{\perp} C$ with the same conditions for the scalars $c_{1}, c_{2}, c_{3}$.

The notion of star-orthogonality was introduced by Hestenes [10]. Let us recall that matrices $A, B \in \mathbb{C}^{n \times m}$ are star-orthogonal, denoted by $A \stackrel{*}{\perp} B$, if $A B^{*}=0$ and $A^{*} B=0$. It is well-known that for $A, B \in C_{n}^{E P}$,

$$
A \stackrel{*}{\perp} B \Leftrightarrow A B=0 \Leftrightarrow B A=0 .
$$

If $A, B$ are hypergeneralized projectors, then $A \stackrel{*}{\perp} B$ or $A B=B A=0$ is sufficient for $A+B$ to be a hypergeneralized projector (see [9]).

Theorem 2.10. Let $c_{1}, c_{2}, c_{3} \in \mathbb{C} \backslash\{0\}, c_{1}^{3}+c_{2}^{3} \neq 0, c_{1}^{3}+c_{3}^{3} \neq 0$. If $A, B, C \in$ $\mathbb{C}^{n \times n}$ are commuting hypergeneralized projectors such that $B C=0$, then the following conditions are equivalent:

(i) $c_{1} A+c_{2} B+c_{3} C$ is nonsingular,

(ii) $B^{3}+C^{3}+A\left(I_{n}-B^{3}-C^{3}\right)$ is nonsingular,

(iii) $\operatorname{rank}\left(A\left(I_{n}-B^{3}-C^{3}\right)\right)=n-(\operatorname{rank}(B)+\operatorname{rank}(C))$. 
Proof. By [5, Corollary 3.9], we have that

$$
B=U\left(B_{1} \oplus 0_{s, s} \oplus 0\right) U^{*}, C=U\left(0_{r, r} \oplus C_{1} \oplus 0\right) U^{*}
$$

where $B_{1} \in \mathbb{C}^{r \times r}, C_{1} \in \mathbb{C}^{s \times s}$ are nonsingular and $U$ is unitary. Since $B^{2}=B^{\dagger}$ and $C^{2}=C^{\dagger}$, we get that $B_{1}^{3}=I_{r}$ and $C_{1}^{3}=I_{s}$. Also, since $A$ commutes with $B$ and $C$, it follows that $A=U\left(A_{1} \oplus A_{2} \oplus A_{3}\right) U^{*}$ where $A_{1}, A_{2}, A_{3}$ are hypergeneralized projectors and $A_{1} B_{1}=B_{1} A_{1}, A_{2} C_{1}=C_{1} A_{2}$.

Now,

$$
c_{1} A+c_{2} B+c_{3} C=U\left(\left(c_{1} A_{1}+c_{2} B_{1}\right) \oplus\left(c_{1} A_{2}+c_{3} C_{1}\right) \oplus c_{1} A_{3}\right) U^{*},
$$

so $c_{1} A+c_{2} B+c_{3} C$ is nonsingular if and only if $c_{1} A_{1}+c_{2} B_{1}, c_{1} A_{2}+c_{3} C_{1}$, and $A_{3}$ are nonsingular. By Proposition 2.3 we get that $c_{1} A_{1} B_{1}^{2}+c_{2} I$ is nonsingular. Now, by $c_{1} A_{1}+c_{2} B_{1}=\left(c_{1} A_{1} B_{1}^{2}+c_{2} I\right) B_{1}$ it follows that $c_{1} A_{1}+c_{2} B_{1}$ is nonsingular. Similarly, we get that $c_{1} A_{2}+c_{3} C_{1}$ is nonsingular. Thus, $c_{1} A+c_{2} B+c_{3} C$ is nonsingular if and only if $A_{3}$ is nonsingular i.e., $B^{3}+C^{3}+A\left(I_{n}-B^{3}-C^{3}\right)$ is nonsingular. Hence, $(i) \Leftrightarrow(i i)$. Also, we have that $A_{3}$ is nonsingular if and only if $\operatorname{rank}\left(A_{3}\right)=n-(r+s)$ which is equivalent with the fact that $\operatorname{rank}\left(A\left(I_{n}-B^{3}-C^{3}\right)\right)=n-(r+s)=$ $n-(\operatorname{rank}(B)+\operatorname{rank}(C))$. So, $(i) \Leftrightarrow(i i i)$.

Remark that from the proof of Theorem 2.10 and Theorem 2.1, if one of the conditions $(i)-($ iii $)$ holds, we get the formula for the inverse of $c_{1} A+c_{2} B+c_{3} C$ :

$$
\begin{aligned}
\left(c_{1} A+c_{2} B+c_{3} C\right)^{-1}= & \frac{1}{c_{1}^{3}+c_{2}^{3}}\left(c_{1}^{2} A^{2} B^{3}-c_{1} c_{2} A B+c_{2}^{2} A^{3} B^{2}\right)+\frac{1}{c_{2}} B^{2}\left(I_{n}-A^{3}\right) \\
& +\frac{1}{c_{1}^{3}+c_{3}^{3}}\left(c_{1}^{2} A^{2} C^{3}-c_{1} c_{3} A C+c_{2}^{2} A^{3} C^{2}\right)+\frac{1}{c_{3}} C^{2}\left(I_{n}-A^{3}\right) \\
& +\frac{1}{c_{1}}\left(B^{3}+C^{3}+A\left(I_{n}-B^{3}-C^{3}\right)\right)^{-1}\left(I_{n}-B^{3}-C^{3}\right),
\end{aligned}
$$

which will be useful later in Theorem 2.11.

In the following theorem, under the assumption that $c_{1}, c_{2}, c_{3} \in \mathbb{C}, c_{1} \neq 0, c_{1}^{3}+$ $c_{2}^{3} \neq 0, c_{1}^{3}+c_{3}^{3} \neq 0$, we show that $c_{1} I_{n}+c_{2} A+c_{3} B$ is nonsingular, in the case when $A, B$ are commuting hypergeneralized projectors such that $A B=0$. Remark that the same theorem holds if we suppose that $A, B$ are generalized projectors such that $A+B \in C_{n}^{G P}$ or when $A, B$ are hypergeneralized projectors such that $A \stackrel{*}{\perp} B$.

Theorem 2.11. Let $c_{1}, c_{2}, c_{3} \in \mathbb{C}, c_{1} \neq 0, c_{1}^{3}+c_{2}^{3} \neq 0, c_{1}^{3}+c_{3}^{3} \neq 0$. If $A, B \in \mathbb{C}^{n \times n}$ are commuting hypergeneralized projectors such that $A B=0$, then $c_{1} I_{n}+c_{2} A+c_{3} B$ is nonsingular and 


$$
\begin{aligned}
& \left(c_{1} I_{n}+c_{2} A+c_{3} B\right)^{-1}=\frac{1}{c_{1}^{3}+c_{2}^{3}}\left(c_{1}^{2} A^{3}-c_{1} c_{2} A+c_{2}^{2} A^{2}\right) \\
& +\frac{1}{c_{1}^{3}+c_{3}^{3}}\left(c_{1}^{2} B^{3}-c_{1} c_{3} B+c_{3}^{2} B^{2}\right)+\frac{1}{c_{1}}\left(I_{n}-A^{3}-B^{3}\right) .
\end{aligned}
$$

Proof. The proof follows by Theorem 2.10 and (2.5).

Corollary 2.12. Let $c_{1}, c_{2}, c_{3} \in \mathbb{C} \backslash\{0\}, c_{1}^{3}+c_{2}^{3} \neq 0, c_{1}^{3}+c_{3}^{3} \neq 0$. If $A, B, C \in \mathbb{C}^{n \times n}$ are commuting hypergeneralized projectors such that $B C=0$, then the invertibility of $c_{1} A+c_{2} B+c_{3} C$ is independent of the choice of the scalars $c_{1}, c_{2}, c_{3}$.

Corollary 2.13. Let $c_{1}, c_{2}, c_{3} \in \mathbb{C} \backslash\{0\}, c_{1}^{3}+c_{2}^{3} \neq 0, c_{1}^{3}+c_{3}^{3} \neq 0$. If $A, B, C \in \mathcal{G}$ such that $B+C \in C_{n}^{G P}$ or $A, B, C \in \mathcal{H}$ such that $B \stackrel{*}{\perp} C$, then the invertibility of $c_{1} A+c_{2} B+c_{3} C$ is independent of the choice of the scalars $c_{1}, c_{2}, c_{3}$.

Acknowledgement. The authors would like to thank the anonymous reviewer for his/her very useful comments that helped to improve the presentation of this paper. Also, we are very grateful to Professor Oskar Maria Baksalary for his useful remarks.

\section{REFERENCES}

[1] O.M. Baksalary. Revisitation of generalized and hypergeneralized projectors. Statistical Inference, Econometric Analysis and Matrix Algebra, B. Schipp and W. Krämer (editors). Springer, Heidelberg, 317-324, 2008.

[2] J.K. Baksalary, O.M. Baksalary, X. Liu, and G. Trenkler. Further results on generalized and hypergeneralized projectors. Linear Algebra Appl., 429:1038-1050, 2008.

[3] J.K. Baksalary, O.M. Baksalary, and J. Groß. On some linear combinations of hypergeneralized projectors. Linear Algebra Appl., 413:264-273, 2006.

[4] A. Ben-Israel and T.N.E. Greville. Generalized Inverses, Theory and Applications. John Wiley \& Sons, New York, 1974.

[5] J. Benítez. Moore-Penrose inverses and commuting elements of $C^{*}$-algebras. J. Math. Anal. Appl., 345:766-770, 2008.

[6] J. Benítez and N. Thome. Characterizations and linear combinations of $k$-generalized projectors. Linear Algebra Appl., 410:150-159, 2005.

[7] S.L. Campbell and C.D. Meyer Jr. Generalized Inverses of Linear Transformations. Pitman, London, 1979.

[8] M.P. Drazin. Natural structures on semigroups with involution. Bull. Amer. Math. Soc., 84:139-141, 1978.

[9] J. Großand G. Trenkler. Generalized and hypergeneralized projectors. Linear Algebra Appl., 264:463-474, 1997.

[10] M.R. Hestenes. Relative hermitian matrices. Pacific J. Math., 11:225-245, 1961.

[11] G.W. Stewart. A note on generalized and hypergeneralized projectors. Linear Algebra Appl., 412:408-411, 2006. 